\title{
Innocent source of humanity
}

\author{
Kyrill Goosseff \\ Rotterdam School of Management, \\ Erasmus Universiteit Rotterdam, Rotterdam, The Netherlands
}

\begin{abstract}
Purpose - To identify the Transcendental Essence of Humanity, the purpose of this paper is to describe in brief what kind of research became possible when the theory of, e.g. autopoiesis, Husserl's Transcendental Consciousness and the theory of Rhodes and Thame came together to form a "transcendental" interview methodology.

Design/methodology/approach - Critical conceptual implications are drawn to form a new research method to explore a de-subjectified inner domain and to search for a possible common essence of humanity. Findings - A Transcendental Emotional Reference was found practically alien to contemporary perspectives. Still, the reference governs the emotional structure of human experience. This different perspective answers basic questions of morality, organization theory and leadership.

Research limitations/implications - The findings of the new research open a new and transparent perspective answering Grey's question: "What is it to be human?" (Grey, p. 47, 2014.) A perspective shedding new light on the humanities. A research limitation is the number of respondents. Still, being transcendental the findings are theoretically valid for all.

Originality/value - The paper is based on a unique research enabling 32+ (ongoing research) respondents to explore their own and universally shared Transcendental domain.
\end{abstract}

Keywords Morality, Autopoiesis, Organization theory, Transcendental consciousness,

Transcendental source of emotions, Veil of innocence

Paper type Conceptual paper

\section{Prologue}

Is there such a thing as the essence of humanity shared by all, and is it moral? The answers become important if they touch upon something real. If real and when found, the answers cannot be metaphysical or based on assumptions alone.

What is real is that life since its origin obeyed a primary principle that pushed life forward into diversity and its development for 3.2bn years. Life created and obeyed the doctrine of the survival of the fittest and it still does. A big part of being the fittest for more evolved species is by being the strongest as a single creature or as a group and to "win" from the competition whereby winners take most if not all. Within humanity this principle can be often recognized, hidden or openly behind military, political, economic and financial interactions. Life's ancient doctrine does not need moral questions to be answered. To be the fittest or the strongest will do to survive and to evolve.

Still, for one species in particular those questions persist as-if being important for thousands of years until today. Through time, within humanity all tribes and cultures live their own (metaphysical) stories, which fade away or change into something else. For some reason time does not have that effect on stories linked to morality or "doing good." For all those years from the Upanishads toward contemporary morality disguised or not in wisdom, in religion, spirituality, mysticism or philosophy a part of humanity hangs on to these stories in a most stubborn way. Stories which not so much oppose the biological doctrine of life, but enrich it with compassion or love. Could these stories express something essentially real of humanity?

(C) Kyrill Goosseff. Published by Emerald Publishing Limited. This article is published under the Creative Commons Attribution (CC BY 4.0) licence. Anyone may reproduce, distribute, translate and create derivative works of this article (for both commercial and non-commercial purposes), subject to full attribution to the original publication and authors. The full terms of this licence may be seen at http://creativecommons.org/licences/by/4.0/legalcode

Received 6 January 2019 Revised 3 June 2019 Accepted 12 September 2019

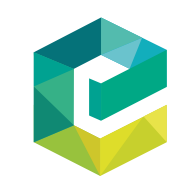

Journal of Organizational Change Management Emerald Publishing Limited 0953-4814 DOI 10.1108/JOCM-01-2019-0006 


\section{Introduction}

MacIntyre (1984/2007) and Grey $(2005,2014)$ have something in common. Both demonstrate separately that a core foundation is missing, but assumed to be silently present underneath the important cluster of theories they analyzed. Moral theories were researched by MacIntyre and organization theories by Grey. In general, theories proposed or brought forward by science or philosophy genuinely support or try to support humanity by the true belief in the validity or plausibility of their theories. A validity established by forming the theories rationally, objectively and based on facts whereby when possible, assumptions are avoided by following approved methodological approaches. To ignore or take for granted a foundational and indispensable aspect in the forming of a theory is highly unusual. Still that is what MacIntyre and Grey have found.

\section{Grey}

Grey gives an account of what is missing in the development of organization theories. Organization theory is not about organizations like the concept "organization" is defined. More or less all definitions of an organization share fundamentally something like this: "A social unit of people that is structured and managed to meet a need or to pursue collective goals." What the genuine aspects of a social unit is, is assumed to be common knowledge. When research produces theories about for instance the species wolf or deer, it faithfully follows all the important aspects of the species from being a social unit toward individual properties relevant for the species. How they live and interacts as individuals among each other within the herd like the behavior of the alpha male all the way down to the last one in the "picking order" and how the herd behaves like a herd relative to its environment. No important aspect is assumed or ignored. Not so much for organization theory.

The study of organizations expressed in academic theory and presented in business schools is mainly about the second part of the definition: structure and managing while strongly reducing or ignoring defining aspects of the first part: a social unit comprised of individual people. Organization theory describes a one-sided aspect of organizations: how to reach and manage efficiency in the broadest sense of the word (Grey, 2014, p. 131). The aspects belonging to what a social-unit is and what its aspects are, are silently assumed. Organization theory describes organizations from a single point of view solely aimed at managers. Grey shows how from Weber to Taylor, from HRT to cultural management, from SMT toward post bureaucracy, change management and new capitalism, they all provide essentially theories and skills geared toward management to find or create the "fittest" (most profitable) organization. Those theories since their origin academically pretend to be based on facts and therefore are presented as rational and objective. According to Grey the managerial understanding of the organizational world embedded in organization theories are given as the only valid representation as if factual based. This is a grave mistake. He shows that organization theory is a "[...] construction [majority shared assumptions], which conveniently excludes almost everything of importance. First and foremost, it excludes the way that it [managerial understanding or theories] is a construction" (Grey, 2014, p. 59, italics added). In principle the concept of organization or business is a reification and organization is a highly abstract example of a construction. For instance, it is impossible to make a scientific valid judgment where the organizational borders actually are or even what they actually are, leading to an ambiguous area of responsibility in the broad interface between organization and its environment.

Grey's (2014) greatest criticism is not the use of reifications which order social realities. His greatest criticism and concern is the important and silently assumed but fundamental assumption founding organization theories about "what it is to be human" (pp. 49-51, p. 59). The "ghost" assumption is used as-if universally valid and makes employees predictable and manageable to fit any mold of any organization. By not mentioning the assumption it 
stays hidden beyond any critique. The rich human diversities of personhood are within organization theory reduced to a single and most simplified generalization with a single function: to be an employee, in service of the organization, compliant to the expectations of management only, within the room the organizational culture dictates. On a superficial level there seems nothing wrong with being compliant to management and their supporting academic theories, except it does not regard a human being to be a member of a social human unit as all defining properties to be human are left out. The "wrongness" is not the job of the employee but the unspoken reduction of personhood to be his or her job. Organizations expect (unconsciously?) individuals to identify with their job and only to be evaluated as such. Only the bravest do not do job identification by staying faithful to their values with little compromises, while staying committed to their job. Job identification and job commitment are two different things (Van Knippenberg and Sleebos, 2006). Job identification may (strongly) reduce the employee's values in order to comply and be part of the prevailing organizational culture (group thinking). Organizations as identities under the contemporary managerial understanding of the organizational world cannot do otherwise than to follow the old biological principle to survive in a competitive world within the rules of law. The old biological principle however basically valid for all life reduces humans to be nothing more than a different species between animals.

Grey shows that whatever essence humanity may have is not part of economic, financial or organization theories. This must be taken literally. There are successful organizations with primarily humane cultures, where the organizational culture is "designed" around the freedom for personhood to meet organizational goals. Not because of any mainstream academic organization theory but of the very out of the box vision of the founder of the company or its successor(s). A clear example is the Brazilian company Semco (Semler, 2001) where layers of managers are missing by participative management or the company Menlo Innovations (Shaer, 2013), where it is hard to find a manager as its responsibilities are not centralized anymore in a single person whereby the organizational structure is designed around people in its center.

\section{MacIntyre}

Conventional science like, e.g. biology, physics or astronomy has an abundance of verifiable empirical facts at its disposal to authorize its theories. What facts or what is real offer moral theories authority? Most leaders and people base ethical decisions on cultural, religious and or institutional moral traditions. That makes what is regarded as "good" in Denmark or California vulnerable to rejection in Pakistan or Texas. Even within one city there is room for contradicting moralities. The suggestion then is that being moral is a kind of "skill" that can be learned from sources embedded in the social environment. As any culture is free to absorb or create metaphysical stories and is free to attach any kind of meaning to facts, social environments are highly subjective by definition. Morality learned from subjective sources will miss by the same process of becoming universal validity. MacIntyre came to the unavoidable conclusion, after scrutinizing the depth and history of Western moral theories that moral philosophy lacks any compelling rational authority (MacIntyre, 1984/2007 pp. 8-11, pp. 9-13). He showed that contemporary participants in the debate on morality assume that their arguments are rational. For MacIntyre moral explanations based on rationality are a grave mistake. Moral rational arguments suggest that objective (impartial) approaches are possible, but they all without exceptions lead in the end to moral inconsistencies, creating disagreements that go on indefinitely: "[...] they [the disagreements] apparently can find no terminus" (MacIntyre, 1984/2007, p. 6). Rational moral judgments warrant themselves:

$[\ldots]$ by referring to some universal rule from which it may be logically derived and can only justify that rule in turn by deriving it from some more general rule or principle [...] since every chain of 
reasoning must be finite [...] such a process of justificatory reasoning must always terminate with the assertion of some rule or principle for which no further reason can be given [...] the utterance of any universal [valid] principle is in the end an subjective expression of the preference of an individual will (MacIntyre, 1984/2007, p. 20 italics added).

This subjectivity will lead to fragmented "universal" principles incompatible among themselves.

MacIntyre agrees with Nietzsche, who he sees as the moral philosopher of the present time. Nietzsche makes clear, posthumous reinforced by Macintyre's research that all moral theories are in the end based on personally chosen assumptions and Nietzsche concludes "[...] if there is nothing to morality as expressions of will, my morality can only be what my will creates [...] I myself must now bring into existence "new tables of what is good," "We, however want to become those we are - human being who are new, unique, incomparable, “[...] who create themselves'” (MacIntyre, 1984/2007, p. 114).

Facts, which in science ground impartial theories and give them rational authority are replaced by moral theories with collective personal assumptions (constructions), a practice shared with the processes of beliefs in metaphysical stories.

Nietzsche could not provide a universal solution with his tables. He could only follow and add the expressions of his own personal "will" to the many ones before him, in the end missing rational authority.

The problem stays: what impartial authority to follow, expressed in "tables" valid for all, which by its impartiality honor simultaneously the freedom needed to develop unique personal will plus honor an individual property, which binds them all by being valid for all. It is the last which has the promise to be or express the essence of humanity being a shared part of everyone but binding humanity as a whole.

If the above promising, binding essence exists, could or should it work in smaller contexts like within organizations? It will answer Greys question of what it means to be human (within organizations). The search requires as a first real impartiality unable to take side, unable to express the unique will of the researcher.

\section{The impartial veil of ignorance}

The use of impartial arguments in defense of morality is to apply the use of an impartial position (free from all personal interest) where the arguments come from. How impossible this seems to be for a unified morality, impartiality at least is pursued on a daily basis by judges, referees, arbiters or mediators in contexts where opposing parties have often strong (conflicting) interests. In all these cases, just rulings are required that will happen when a genuine impartial position is accepted. What is real in the concept of impartiality?

We take it for granted that language is not real compared of how empirical facts are experienced and regarded as real. The realness of language is based on its function of signifying to what is (accepted) as real. As language so easily fulfills that function, language itself becomes for us in a natural way as valid and as real as the signified itself. This is rather "innocent" with existing real (objective) concepts, e.g. objects or trivial constructions as "cold" or "future," A little bit strange is that the accepted realness of language authorizes to make unreal concepts which it refers to as also real as language itself, as if being a legitimate part of material realities. For instance, cold is a meaning and not a fact. Cold cannot be a real property of the signified. Also, the concept "tomorrow" only exists and functions today, being a part of today contradicts the meaning of tomorrow. Still it is innocent. Concepts like "stranger" or "poison" applied to ideas are much less innocent. Impartial means literally not-partial as in not biased, it always means something else is real and presented instead. "I don't see a tiger." may express the experience of seeing an empty cage or a painting of a yellow beast in limbo or a million different other things that can be (self) verified. Then, what is real in the concept of being impartial, but not described by negations? Dictionary descriptions turn to synonyms 
like objective but also "fair" and "just" without explanation, but also without exceptions. Being neutral or objective equals the perspective of impartiality, the logic behind those synonyms is trivial. What is the logic behind the synonym's justness and fairness? They are not self-explanatory. If one walks in a forest with dangerous animals and accepts the forest to be neutral and impartial, overlooking a valley to be crossed, what fairness and justness can be expected during the journey? It must be something humanness, which the forest cannot provide. Fairness, justness or righteousness are the effects of humans being impartial. Only an accepted genuine impartial position may awaken something universally humanness like fairness or justness or mutual respect within opposing parties to find a common solution.

John Rawls (1971/2009) turned to impartiality in his book A Theory of Justice, in which he advocates a theory for just income distribution as part of a just society. His theory has a "golden lining," as-if its moral ground stands apart from his personal assumptions (personal will) as the author. He begins with a "veil of ignorance" obtained under a hypothetical meeting of different people:

I assume that the parties are situated behind a veil of ignorance [...] First of all, no one knows his place in society [...] social status [...] fortune - abilities, his intelligence and strength [...] his conception of the good [...] special features of his psychology [...] particular circumstances of their own societies - [...] It is taken for granted, however, that they know general facts about human society [...][known] principles of economy theory; laws of human psychology (Rawls, 1971, pp. 136-137).

When forgetting all personal properties one indeed de-subjectifies oneself and occupies a true impersonal "original" position necessary to start moral discussions with the promise of authoritative outcomes. But the book attracted next to high praise also (severe) criticism. First, Rawls' original position is not real but a hypothesized one and for opposing ideologies (libertarians, communitarians) easy to deflect. A second, but important one is what are the "taken for granted" known principles of economy and psychology? Two complex contemporary important topics, both missing an accepted universal theory or even point of view. A third point is that for Rawls even when true impartiality is reached (hypothesis) the impartial agents in question become pure rational agents. Rationality "pur sang" however is unfit to decide between values. Even when truly forgetting one's personal attributes as described above to enter the veil of ignorance, one does not become a pure rational being as was assumed. A question like "What is the primary most important aspect of a good friend?" will give different to non-compatible answers even among the ones under the veil. Different values and their relative importance cannot be determined by logic alone. Rational approaches will be influenced and actually need unconscious complexities of meaning, emotions and intuition as soon as questions of values become real. Human conscience cannot be fully understood in the grand scheme of things in a pure rational way. In total, $3.2 \mathrm{bn}$ years of successful biological life show that pure rationally conscience is irrelevant for life to be highly effective. Only a part of humanity for some unknown reason adds conscience to the biological doctrine. A pure rational emotionless agent will much better represent for instance a high order artificial intelligence robotic "species," but will be out of touch with humanity.

\section{The veil of innocence}

To avoid the associated difficulties of a hypothetical veil of ignorance, a different kind of veil is needed. The "veil of innocence" named after Rawls' veil of ignorance makes a true nonhypothetical impartial position possible, but functions also as a start to find a humane impartial position suited to represent humanity. Thus, next to a true impartial rationality as a property of consciousness, the integration of a next important property linked to emotions is needed as well. The proposition is that only together, integrated somehow the essence of humanity maybe found. The veil of innocence stands for a rather new and radical interview 
method (Goosseff, 2015). The method enables people to enter a full impartial state of consciousness equaling Husserlian Transcendental Consciousness. Transcendental here refers in a Kantian way to "a priori to the world," like the ability to see is a priori to what can be seen. In other words, the ability to see exists independent from the world and comes before it.

Husserl described Transcendental Consciousness as: "[...] the most general, subjectivity, which makes the world and its 'souls,' and confirms them" Husserl (1927, p. 8 italics and underlining added). "Most general subjectivity" can easily be replaced by universal subjectivity shared by all. His "Transcendental Consciousness" is a real experience. It resembles the impartial agent under Rawls' veil of ignorance, but not as a hypothesis.

Husserl:

I learn to survey transcendental experience. I am no longer interested in my own existence. [...] My psychological experiences, perceptions, imaginations and the like remain in form and content what they were, but I see them as "structures" now, for I am face to face at last with the ultimate structure [the ego] of consciousness (Husserl, 1927 p. 8 underline and italics added).

"I am no longer interested in my own existence" must be understood within the context what Husserl needed. He was purely interested in what is truly real, in that what is not an assumption/construction. Most if not all people consider their personality or identity or their ego (even as a researcher) as real enough, but not for Husserl. Husserl could have accepted that position too and then leave it be, but by following in a sense Descartes' method to doubt the validity of all knowledge, Husserl, by putting the validity of knowledge in symbolic brackets and then disregards them as-if not proven yet, went further than Descartes. He also found it possible to experience himself out of any knowledge about himself. Descartes did not discover his personality to be a construction. Husserl realized his ego was a very real "ultimate construction made by himself, [...] I am face to face at last with the ultimate structure of [my Transcendental] consciousness," In the end Husserl experienced himself to be a transcendental consciousness that could not be bracketed or invalidated anymore. This transcendental state is not knowledge, but a pure experience of a-thing-in-itself. This state of consciousness is not a topic of interest in traditional or contemporary psychology and cannot be reached with existing academic methods nor is it a natural state to be in. Although Husserlian transcendental consciousness will be a necessary condition to start the search for the essence of humanity, it is not enough.

During his research Husserl also found that within one's own mind, one (in present ego) can contact his own ego "[...] its past ego, even though the latter is precisely no longer present: it can have a dialogue with it [...] (Husserl, $1970 § 50$ p. 72, italics added). Note that Husserl does not say: "I can have a dialogue with it," The "I am" is only free of the ego as Transcendental Consciousness, which is not necessary to contact part(s) of the ego. The two biologists Maturana and Varela (1972/1980) being the researchers behind autopoiesis theory mention self-interaction in their theory of Autopoiesis described in the book "Autopoiesis and Cognition":

The ability of the nervous system [neo-cortex] to interact with its own states, as if these were independent entities [...] (Maturana and Varela, 1980, pp. 25-26, italics added).

Communicating internally although non-perceivable for observers is acknowledged by, e.g. Husserl, by Maturana and Varela, by Voice Dialogue of Stone \& Stone (1989), the studies of Archer (2007), and above all is easily self-verifiable. In that sense internal dialogue is not metaphysical, but a real verifiable fact within just like experiencing emotions. Stone \& Stone showed that the ego cannot reliably be expressed as a single unity or by one voice. They found dozens of voices able to contradict each other. Archer (2007) brings forth the "normality" of hearing internal voices or internal dialogue and she places them outside the psychopathology of contemporary mainstream (clinical) psychology and psychiatry 
(Larøi et al., 2012). The conclusion is justified that under the right conditions an internal, but real impartial interview may be possible between [Husserl's] Transcendental Consciousness and a further "transcendental part of the unconscious" linked to emotions.

Goosseff's (2015) interview technique (veil of innocence) builds upon Stone and Stone and incorporated Rhodes and Thames (1988), who concluded how the vast complexities (the many voices) of a person's ego to handle information can be reduced to "only" three functions (functional categories) each having its own voice: the Realist, the Critic and the Creative, who together like an internal management board of three constitute the whole ego. Rhodes and Thame avoided stereotyping among people by assigning colors to their respective functions:

Everything you can possibly intend to do with your thinking is found within the one simple picture in Figure $1[\ldots]$ Green is the code for the unknown, the as yet unrealized future [...] Green thinking is the fountain of creativity [...] Red is the code for what is true, what is already known $[\ldots]$ this is the thinking that finds out, seeks out information [...] It collects facts and figures [...] Blue is the code for coming to judgment, it requires personal values and beliefs (Rhodes and Thame, 1988, p. 38).

To enable participants to become egoless (an ancient goal of meditation) in a single interview or in other words to learn how to associate themselves with (Husserl's) transcendental consciousness, they simultaneously dissociate themselves (temporary) from their ego. However, thousands of years of meditating practices show how difficult this is and how impossible to overcome for many practitioners. Meditation especially for novices is a struggle and needs a lot of patience and discipline. Historical and contemporary meditation practices rely on a common, simple and most basic assumption: the ego is not part of the will of the meditating agent, it is rather an opponent to be silenced (e.g. Krishnamurti, 1966).

The assumption confirms the incompatible polarity between an a priori (spiritual) consciousness and a worldly ego. The incompatibility will result in a "fight" when one wants to still or conquer the other without its consent. We should not underestimate the conclusion

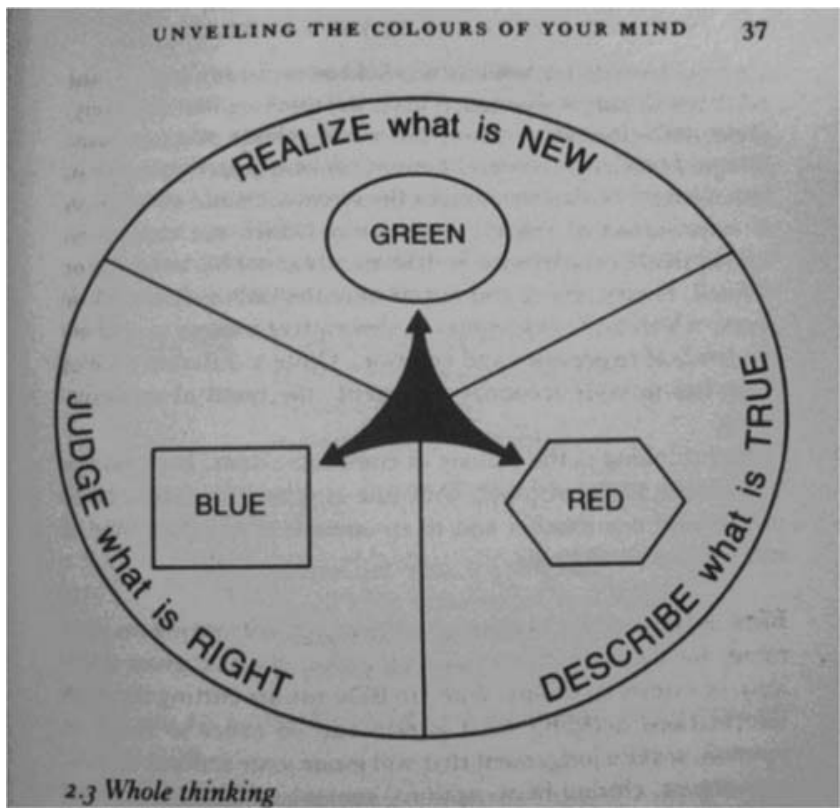

Figure 1. Colors of your mind 
made in autopoietic theory: "The ability of the nervous system [neo-cortex] to interact with its own states, as if these were independent entities [...]" The power of these as-if independent "entities to influence or have a voice is real.

The interview method therefore is based on the choice to inverse the ancient assumption about the ego (Goosseff, 2015, pp. 144-156) and to approach it as an "ally" or even "friend "and not as an "opponent," The ego in the form of a management board of three is invited to make the veil of innocence happen following similar methods as used by Stone \& Stone (1989). To establish the ego's silent cooperation as indispensable is a radical new approach. The three members are separately offered a contract to internally not only allow the rise of a transcendental position, but also to allow the impartial agent of consciousness to further explore its transcendental substance. In exchange for accepting the contract, as part of the contract itself, the board and not the exploring agent decides what to do with the outcome of the transcendental survey. Acknowledging the contract by understanding there is nothing to lose by accepting it, the ego silences itself freeing transcendental consciousness, but within the context of the experiment. There is no struggle, no fight and the transition is within seconds. In two of the cases out of 32, ego did interfere at some point, but for the exploring agent contamination is very easy to detect from within his transcendental silence. Respectfully referring the interruptions to the contract, freed the way to continue. Interviewees in their transcendental state discover another transcendental entity under the veil of innocence: the innate transcendental source of emotions (Goosseff, 2015 pp. 125-128, $130,133)$ not to be confused with emotions themselves. The infinite number of rational and irrational possible links between thoughts, meaning and emotions makes it practically impossible to find a unified view of the essence of humanity or genuine human nature beyond emotional diversity. Rather the opposite is present, which suggests that there is no unifying essence. To skip the rich category of emotions altogether shifts the attention to their common source.

The Transcendental source of emotions is found and experienced by all respondents who participated in the research, whereby the source could be contacted in a natural way. During this internal impartial "interview" between transcendental consciousness and transcendental source of emotions the practice of "member check" or "respondent validation," a common qualitative research method was internally applied multiple times in order to secure the agent's confidence about the source of the received answers (Goosseff, 2015 pp. 147-148). Under the veil of innocence, the search began to answer the overall question "How does the Source of emotions know or what reference is followed in order to "generate" positive or negative emotions as internal responses to "triggers" and "what are those triggers?" The very fact that most if not all people do not consider their emotions to be blind, random and unrelated to thoughts or worldly events, already suggests that a kind of rational reference must exist somewhere in the background. It does and is easily methodically self-verifiable (Goosseff, 2015, p. 149)

\section{The outcome of the veil of innocence I, self-referentiality}

The "raw" outcome of the internal interview between transcendental consciousness and his "counterpart" the transcendental source of emotions (Goosseff, 2015, pp. 157-160) was this: any idea (read meaning) believed, allowing the agent (the "owner" of the idea) to be genuine himself in a context or to develop and grow his genuine self into experience, will be accompanied with a positive emotion from his emotional source. The reverse is equally true. Ideas that hinder growth and development or hinder an already developed genuine part to be expressed in the present, will be accompanied with negative emotions. The rational or reason behind the ideas are regarded as irrelevant by the source, how important or essential they were for consciousness to construct them. Next, ideas that are accepted to help others or to hinder them or not helping them, seem to follow that same reference too. To have 
feelings about self or others following the same identical reference was noticed by researcher and respondents and presented to the transcendental source. Its explanation will be dealt with a little further in the text in the outcome II.

The outcomes, were identical for all participating respondents. The interactions through emotions between emotional source and consciousness' ideas obey a transcendental reference, which superficially seemed twofold: one for self and one for the outside world including others. The single reference comes out of self (SelfReference). Self with a capital S, the "S" stands for to be genuinely oneself (interacting) in some context, as there is always a context. SelfReference follows literally the metaphor of a (rose) seed. Being genuinely oneself needs a critical examination, what does it accurately mean?

If we look at a rose seed, comparable to a full rosebush of colorful flowers and their fragrance, we see nothing of the latter. Yet, all of that is present, but hidden in the potential of the rose, waiting in the seed to be expressed. The seed depends on a mechanism to realize its potential over time. Its essential mechanism is known. A gene-based species determined "blueprint" (organization) regulates biological processes of growth and development in a rather mechanical way. The rose grows and develops and eventually expresses its full potential under the right external conditions. Right is what the rose really needs in order to develop its potential. Principally, there is no error from within; the internal processes are genuine and error free. Very rare a malformation occurs, like for instance a cow with two heads. The rose during its processes of development and growth is in a natural way its "genuine-self." All internal "life-energy" is geared toward and circles around self-growth and -development through self-expression. For the rose there is no difference between self and Self, they are one.

For humans it is so much harder and close to impossible for many to be their Self most of the time. What the rose is fully missing, is abundantly present for humans: the unconditional freedom to make up one's own mind of what is important and meaningful to oneself. A significant disadvantage of this freedom is that all meaning-making-processes are vulnerable to influences from outside based on errors, and mistakes are easily made, especially by the very young. Hans Christian Andersen and Letts (1995) famous tale of the swan, which teaches itself (self-description) to be an ugly duckling is a perfect example of making an important error in meaning. In the swan's potential (genuine nature) there is no duck waiting.

Back to the veil of innocence, the SelfReference found, determines the two categories positive and negative emotions by applying a simple principle, compliant to the biological theory of autopoiesis and simultaneously transcending it. The reference fully follows the "organizational closure" principles: self-referentiality, self-creation and autonomy, which is valid for all life and central nervous systems in particular (Maturana and Varela, 1980):

A system is organizationally closed if all its possible states of activity must always lead to or generate further activity within itself [...] and have no inputs and outputs (Mingers, 1995, p. 32 italics added).

The "[...] have no inputs and outputs" explains that all internal processes rely in a circular way to each other albeit that many are triggered by external triggers outside the domain of organizational closure. That makes the internal logic within organizational closure also circular, in the end referring to itself. The "[...] have no inputs and outputs" underline the fully solitary unique nature of the three principles (self-referentiality, self-creation and autonomy), they are free from the world. Fundamentally it means that the living system takes itself as the only reference independent of the world to govern all internal (circular) processes and structures including the important meaning making processes of humans. Many independent internal processes however can be triggered by the world by triggering the many senses. The theory of autopoiesis cannot be avoided here in order to realize how deeply autopoiesis theory explains not only the physiological root of Life and how CNS and 
brain constitute reality, but also how existentially the most core autopoietic principle "organizational closure" reflects the processes found within the transcendental domain. Autopoiesis makes clear that living organisms cannot absorb the meaning of the world which for autopoiesis is a vast dynamic context of meaningless triggers.

The organizational closure principle: self referentiality is present in the fact that the emotional reference has its (potential) genuine Transcendental Self (not to confuse with its consciousness) as its "template" or mold.

The SelfReference governing emotions, deeply centered in one's own potential, links any error in description of Self to a negative emotion or at least to the absence of well-being. An error is a description mismatching Self. As such, this does not sound illogical. If I force my feet into too small or too big shoes (mismatching), when walking, my feet will send me feelings of discomfort or even pain. Untouched by the reason behind the choice for the shoes, in order to be and operate faithfully to what they are, feet demand fitting shoes. The experience of being genuinely oneSelf is the effect of having faith in a matching Self-description, which faithfully matches a relevant part of one's own transcendental potential. Such descriptions allow Self to come out of its potential and to be experienced in its relevant corresponding contexts. If someone beliefs something about self, it implies simultaneously (an) embedded relation(s) with (a) corresponding context(s) and vice versa. It is more or less the relation with the corresponding context that proofs to the agent that the (self-)description is justified, genuine and true. The relation will be addressed under "Circular Logic." A Self-matching description will be accompanied by a positive emotion or the experience of well-being. A positive emotion allows Self to develop or to be in a particular context unhindered by wrong beliefs. Well-being for practical purposes is defined here as a positive emotion, which might be or not under the threshold of awareness. A negative feeling under the threshold, although not in awareness is still not positive despite how it is regarded by consciousness. It is important to realize that the effect of emotions on the person is not determined by the emotions themselves. What they mean to the person, the meaning they are given by consciousness does. The intensity of the feeling experienced, is linked to the relative importance given to the meaning and results in how up front or repressed the feeling will be felt.

The principle self-creation is expressed by self-constructed meanings as no other meanings exists:

Neither information nor truth should be confused with meaning. Meaning is the [personal] understanding that is generated within us, whatever the originator of the symbol [in language] intends [...] Information is neutral, meaning is affective, that is, involves emotion (Jackson N., Carter P., 2000, p. 21).

Only meaning triggers the source of emotions to respond with emotions, not what triggers the meaning. Meaning is an interwoven response to two questions in a fixed sequence, which internally are realized as one question: "What is it [...]?" and "[...] what does it mean to me?" whereby the second answer provides the necessary meaning and cannot be avoided. The first question may not even be answered fully before the second one is already in process. The answer "I don't know (yet) what it means to me" is meaningful by itself and tags the meaning of a kind of "?" to "it." The second answers providing meaning determine the nature of emotions or motivation and make behavioral choices possible, but within the contexts answered by the first question. For Life the second question is the only relevant one. The first one triggered is a necessary precondition serving the second. The adjectives given to the world and self roughly give away the answers of the second questions. Adjectives above or under the threshold of awareness named or kept silent, are ever present. Thoughts and for instance physical pain are internal triggers, which may trigger a correspondent (meta-) meaning. Like in meditations to acquire an inner sense of peace, the 
advice is often given to accept all emerging thoughts to be meaningless not worth any attention or the positively interpreted muscle pain (no pain, no gain) the next day after a good workout.

The second type answers also explain most, but not all differences between people's egos and their corresponding behaviors (Goosseff 2015, pp. 111-123). In short, adjectives giving away the answers of the second questions linked to the contextual answers of the first questions, do not explain the world at all, but their perceivers:

It is safe to say that experience without meaning cannot exist and language without adjectives does not describe experience. That makes (scientific) objectivity a linguistic construct, a regulative idea (Goosseff, 2014, p. 706).

The autonomy of the process emotion vs meaning is shown by its functional independence from the outside world including one's own body and even one's own thoughts. It is full dependent on its template, its potential or genuine Self. SelfReference as part of organizational closure, determining the nature of emotions is about meaning and not about what triggers the meaning, they are separate and not the same. The discovered Transcendental SelfReference reframes emotions as guides to consciousness to help it choose the right Self-descriptions with the right meaning that matches one's true potential. The reference behind emotions explains meanings including the ones about the world to be submissive to that ultimate goal: the discovery of true Self "transcendentally" interconnected with others, patiently waiting to be expressed and developed further.

Joy (not to be confused with pleasure), grieve, anger and fear are the four basic emotions and they have their own specific functional meaning. Joy or under the threshold of awareness well-being is explained earlier and no warning is involved. The negative feelings are all warnings and fall outside the scope of the article, they (emotions) deserve an article by themselves. But briefly, the four basic emotions are like four basic colors, which in combinations with the many different states of the body and the many meaning-relations with contexts, offer a pallet of hundreds of different color experiences like love, awe, guilt, boredom feelings of revenge and so on. A well-prepared steak under a green lamp will taste very different for many people, or getting flowers or a kiss will trigger different feelings depending on the giver and the context. The meaning of the context slips into the meaning of the actual trigger and changes its emotional experience in infinite gradual ways. Mostly the experience will suggest one meaning is to be experienced as a property of the most relevant actual trigger. Another example is an obvious rational story told by an unpopular narrator will be often disregarded.

An important conclusion must be made, which directly comes out of autopoiesis theory and is best explained by the concept of circular logic (CL) applied to language. CL states that every meaningful external description uttered or written down explains in the end only the author. A meaningful utterance or text is defined here by including meaning like adjectives, mostly found in stories or narratives and rarely in objective descriptions. The sentence "Tigers are dangerous animals" reveals not the tiger, but the speaker who must admit: "I don't know how to guarantee my safety when I meet a tiger in the flesh." Successful big cat tamers in a circus will have other ideas like successful drivers will have for all kinds of traffic whereby mistakes can be more deadly than a tiger is able to be. But in all cases the justification of external adjectives is rarely experienced as properties of self, but are experienced as being true properties of the perceived. The tiger is entitled to the response: "How can $I$ be, what you cannot guarantee?" like the stranger may reply with: "I am not a stranger to myself, how can $I$ be, what you don't know?" We can already deduce from this that for instance fear is not a signal to proof an external danger, but an internal one. Fear signals a belief which hinders or even forbids to experience Self in a context. As rational as it seems to understand fear for tigers, the fear does not address the tiger, but the unspoken 
statistical impossibility to keep yourself safe in meeting one in the flesh. As most men have no clue how to do that, they overestimate their own ability to be safe. However, to feel fear standing before a tiger in a solid cage or fear of speaking in public, they underestimate their ability. In both cases fear signifies an unjust belief about Self, hindering Self to be experienced. Accepting danger, while simultaneously accepting the truth of one's own present abilities produces no fear, while acknowledging the danger. We will discuss Levinas' "innocence of humanity" in a moment where he finds innocence in the eyes of the condemned, not fear. The real truth accepted of one's own capability will lead to avoiding contexts which are dangerous. In that case the conclusion to avoid does not need fear. Or the choice is to develop capabilities and with self-confidence reframe dangers ( automatically) into challenges like the tiger tamer or car driver once did.

CL shows all meanings experienced of the world and its events (contexts) including beauty, reflect and explain in the end their maker projected unto the world making it their world. Finally, because of this, all meanings are one sided relational. It is easy to see how adjectives externally given, determine or highly influence the nature of interaction. Meanings do not explain the world but do, explain and often predict their owners how they will interact with their world. The experienced world is a relational world compliant to the personality of the ego, regardless of how s/he feels, regardless if s/he is wrong or not, the compliant world is a stubborn given. Organizational closure makes possible that meanings between persons do overlap often fully in language, but only to a reduced of strongly reduced degree in experience.

So, the important given of the world is reframed by organizational closure as necessary, but meaningless contextual triggers to enable in general, life to develop, to grow and eventually to evolve according to its own nature. The species humans in particular has the least amount of genetic instincts to determine a part of its described nature being the sum of all meanings experienced. For humanity, freedom to make up his own mind does not include freedom to be genuinely oneSelf (Being), unless the corresponding meaning is discovered and accepted in a joyful way or at least in well-being. If not, a kind of false version of Self (self) will be negatively experienced as if real.

\section{The outcome of the veil of innocence II, the essence of humanity}

SelfReference governing emotions, does not directly explain the nature of emotions about others. CL shows within organizational closure that positive or negative adjectives given to other people do not primarily explain others. If we add emotions to CL the negative emotions still suggest that some belief is present to block or hinder the experience of Self in the relationship with the other(s). What is the error believed?

The dilemma was reflected to and solved, by the Transcendental Source of Emotions. Its solution contradicts or even destroys the base of the ego, but only within the unconscious Transcendental Domain. Because of projection, consciousness distances itself from what it has made. In this perceivable distance by the senses the ego regards itself as being different, independent and apart of all meanings it senses in the world and particular those of others. To protect and maintain the ego's identity as a "standalone" existence this "distance" becomes existentially needed to define ego, except in all cases of love.

However, on a Transcendental Level in the unconscious beyond the subjective layers of the individual ego (total sum of personal meanings), the distance and distinction between Self and Other is denied by Self. The Source of emotions for all respondents reflected literally: "I don't make any distinction between myself and (any) others" (Goosseff, 2015, p. 159). Apparently in this dimension existential differences and distances between people cannot be applied. Self appears to be deeply related to itself as it exists in others. This given surprised all respondents as well as the researcher. It is rather blunt. Still, it does perfectly explain to have feelings about self or others following the same identical reference. 
The Reference is followed for both because the distinction between Self and Other, which introduces differences, does not exist in the Transcendental Domain. This equals the acting out of subject-less love meaning unconditional Love or Transcendental Love with a capital L. Love in its essence is to deny any distance (as if being one) between consciousness and the subject of love, while accepting the "other" as part of oneself reframing any (physical) distance as a happy connection. The most intense connection is based on its indestructibility. No conditions can possibly exist to lower or to end this Love making it unconditional, but also impartial. Transcendental Impartial Interconnectedness revealed by the Reference unifies all human beings as equally worth and valid members of one family despite their subjective differences. A family which embraces each member on their level of Being in whatever phase of development they may find themselves to be, in whatever way they express their myriad possible unique subjective ego's, right or wrong. Transcendental Self is not blind to sin, but acknowledges the illusionary root of sin for what it is: the belief and mistaken faith in wrong information as in illusion by not describing any truth about the genuine nature of the mistaken believer. Mistakes in descriptions do not describe what is real and what is real does not need descriptions to be real. Consciousness needs real descriptions in order to allow what is real in experience. Mistakes do not negate sinful thought and their expressions in behaviors, they do exist, but do not prove existence. Experiencing mistakes or believing the mistakes of others will hurt and corresponding behaviors may hurt others. But no mistake proofs a sinner, but may lead to sinful behavior. It is theoretically impossible to make mistakes on purpose. Every amoral motivation going against SelfReference, in the end serves a major belief, innocently accepted to be true, the only reason why it is accepted by not being recognized as a mistake. The innocence will also destroy the dark side of humanity. There is no dark side. There is a real side expressed and a real side forbidden replaced by illusions believed, simulating a dark inner reality, which is felt. All amoral thoughts and behavior, all amoral aspects of cultures do not express human nature, but the freedom of its innocence to be mistaken. Levinas did add "innocence" as an essential property of humanity. Its innocence can be experienced in the eyes of others (not in their pictures or in mirrors), unfortunately only possible for most to detect in an extreme context, in the eyes of the ones just before their execution:

The infinite paralyses [ego's] power by its infinite resistance to murder, which, firm and insurmountable, gleams in the face of the Other, in the total nudity of his defenceless eyes, in the nudity of the absolute openness of the Transcendent (Levinas, 1969 p. 199).

Levinas did not need to be in his Transcendental Consciousness. His ego was bypassed, "paralyzed" as a book of meanings without a reader , opening the door to allow the meeting of Selves triggered by the eyes of the Transcendental Other. An experience made possible because the Other's ego became meaningless after accepting its immanent death by execution. Levinas met the real representative of the innocent egoless Essence of Humanity.

Still, sinful behaviors need to be addressed and stopped when necessary, but sinners are not acknowledged by Self. The blunt distinction between Self and Other not made transcends the individual Emotional Reference within organizational closure. The acceptance of the Other simultaneously destroys the solipsistic confinement of organizational closure.

How does this forgiving essence of humanity, turns out in daily practice? In daily practice it means that personal beliefs about others will be just as hurtful or joyful and everything in between as if they were beliefs about self. Only the intensity of experiencing is under free will because the importance of the meaning constructed is part of the construction itself.

\section{The innocent essence of humanity answers Grey and MacIntyre}

Grey's main criticism on organization and economic theories is the full reduction of a representative of humanity to be an employee or to follow a "representative agent" even 
when tremendously reduced by an algorithm. What it is to be human is fully deleted without a trace. MacIntyre seemingly did find the opposite in moral theories where in a highly fragmented way subjective (group)criteria fight for dominance to define a cultural version of what it is to be human. How can the experience of the respondents "solve" the criticism of both Grey and MacIntyre and others?

When one wants to take the Self of others into account in any kind of relationship, one will acknowledge simultaneously one's own Self. They go together like it takes one to know one. The relationship will awaken corresponding virtues as a gift of Self to both consciousness. One conclusion in order to walk the road to Self is this:

Do unto others as you [deep in your heart long for] have them do unto you.

The wise rule sums it all up and is known for two thousand years, so what is new? The little addition "deep in your heart" is to make it easier to touch upon real values as opposed to cultural ones. Although an advice where hell cannot enter, it has no rational authority opposed by the biological rule of the fittest. It falls within the ones MacIntyre accounted for. Still, SelfReference is a full moral reference. It defines the essence of a universal morality with a capital M. It does not redefine morality, it gives an old two thousand years old reference authority. Those deep in your heart transcendental values are now proven to be real at least for the randomly chosen $32+$ respondents. The Transcendental SelfReference experienced in a Husserlian/Kantian Transcendental domain makes the Self theoretically real for all mankind. SelfReference is a transcendental and autopoietic universal given.

How does a $2 \mathrm{~K}$ years old Transcendental advice handle parties serving a common goal like organizations? Well, how does a $2 \mathrm{~K}$ transcendental advice handle opposing parties, opposed to the core? If it will work for the latter, it will work for the first.

Parties without conflict, but with different (sub)cultures or status opposing each other (fundamentally) by having needs mismatching the needs of the opposing party, will follow an impartial rule albeit often imposed by an impartial referee, as in games and sports. Essential for referees is their impartiality, making the rule of "the game" transparently valid for everyone without exceptions. Players are accepted and respected as human players despite their personalities, color, background (culture), language or status in the team to meet each other under the same rules while the game itself is accepted by a larger community. A good sporting game is principally a Moral one following without knowing Transcendental Values. Applying this given for organization theory is not a dream and Grey's objection to reduce personhood will disappear. Accepting Transcendental Authority of Selves will un-reduce economical and business theories by humanizing them in their application with the infusion of Morality. A Morality that will embrace any kind of industry or organization to be unique and functional within its Moral domain, ending any nonagreed asymmetric rules of the "game". A unified Moral culture based on what humans are, cannot accept the denial of their SelfReference. There is no more "Where ignorance is bliss, 'Tis folly to be wise."' (Gray, 1747). To introduce what it is to be human into organization or economic theories is to authorize Morality. Its leaders and managers will behaviorally reflect in a genuine way the moral guidelines mentioned in e.g. the "The MBA OATH" (Anderson and Escher, 2010), by realizing leaders are serving primarily themselves in serving others. Externally, the organizational environment (society, world) will also be served by being faithful to what it means to be human, expressed in goals and missions compliant for instance to the "green" Cradle to Cradle concept of McDonough and Braungart.

Still, a-Moral beliefs, under the rationalization of functionality will exist as a consequence of the freedom of will. A superior world free of mistakes cannot exist with the existence of free will. Hindering beliefs denying positive attributes will be made, unavoidably in one phase or another for all to experience. The call mistakes make however is for correction, not separation, based on the understanding of the living-innocence behind all mistakes. 
An innocence, which needs guidance to learn to respect and to allow its own Self in others, or in African Ubuntu: "umntu ngumntu ngabanye" (what makes us human is our recognition of the humanity in others) (Luhabe, 2002).

Finally

Notwithstanding, all information false or true like this text is meaningless through the organizational closure within the reader providing total freedom to the reader to make up his own mind about it. All what is real and meaningful cannot force itself upon the mind. Transcendental Love forbids it. Still, if we can, McCloskey states:

If we can do all this ethical reinvestment, we can avoid repeating the slaughters and lesser sadnesses of the twentieth [+] century (McCloskey, 2006, p. 32).

\section{References}

Andersen, H.C. and Letts, K. (1995), The Ugly Duckling, IE Clark Publications, Schulenburg, TX.

Anderson, M. and Escher, P. (2010), The MBA Oath: Setting a Higher Standard for Business Leaders, Penguin, New York, NY.

Archer, M.S. (2007), Making Our Way through the World: Human Reflexivity and Social Mobility, Cambridge University Press.

Goosseff, K.A. (2014), "Only narratives can reflect the experience of objectivity: effective persuasion”, Journal of Organizational Change Management, Vol. 27 No. 5, pp. 703-709.

Goosseff, K.A. (2015), “Transcendental morality and organizational leadership”, available at: https:// hdl.handle.net/1765/79619 (accessed December 11, 2018).

Gray, T. (1747), An ode on a Distant Prospect of Eton College, R. Dodsley and sold, London, p. 51.

Grey, C. (2014), A Very Short Fairly Interesting and Reasonably Cheap Book About Studying Organizations, Sage, London.

Husserl, E. (1927), Phenomenology Encyclopedia Britannica article as published, Northwestern University Press, Evanston, IL.

Husserl, E. (1970), The Crisis of European Sciences and Transcendental Phenomenology * An Introduction to Phenomenology $\$ 22-\S 64$, pp. $84-223$ by *, Northwestern University Press, Evanston, IL.

Jackson, N. and Carter, P. (2000), Rethinking Organisational Behavior: A Poststructuralist Framework, Pearson Education, North York.

Krishnamurti, J. (1966), "Saanen 7th Public Talk 24th July 1966”, available at: www.jiddu-krishnamurti. net/en/1966/1966-07-24-jiddu-krishnamurti-7th-public-talk (accessed December 11, 2018).

Larøi, F., Sommer, I.E., Blom, J.D., Fernyhough, C., Ffytche, D.H., Hugdahl, K., Johns, L.C., McCarthy-Jones, S., Preti, A., Raballo, A. and Slotema, C.W. (2012), "The characteristic features of auditory verbal hallucinations in clinical and nonclinical groups: state-of-the-art overview and future directions", Schizophrenia Bulletin, Vol. 38 No. 4, pp. 724-733.

Levinas, E. (1969), Totality and Infinity: An Essay on Exteriority, available at: http://chungsoolee.com/ files/Totality_and_Infinity_Whole_book_Word_PDF_2012-03-14.pdf

Luhabe, W. (2002), Defining Moments: Experiences of Black Executives in South Africa's Workplace, University of Kwazulu Natal Press, Kwazulu.

McCloskey, D.N. (2006), The BourgeoisVirtues: Ethics for an Age of Commerce, The university of Chicago Press, Chicago, IL.

MacIntyre, A. (1984/2007), After Virtue, University of Notre Dame Press, NotreDame.

Maturana, H.R. and Varela, F.J. (1980*), Autopoiesis and Cognition, D. Reidel Publishing, Dordrecht, *English translation of "De Máquinas y Seres Vivos" (1972).

Mingers, J. (1995), Self-producing Systems: Implications and Applications of Autopoiesis, Plenum Press, NY. 
Rawls, J. (1971/2009), A Theory of Justice, Revised ed., Harvard University Press and Oxford University Press, Cambridge, MA.

Rhodes, J. and Thame, S. (1988), The Colours of Your Mind, HarperCollins, Glasgow.

Semler, R. (2001), Maverick!: The Success Story Behind the World's Most Unusual Workplace, Random House.

Shaer, M. (2013), “The Boss Stops Here”, New York Magazine, available at: http://nymag.com/news/ features/bossless-jobs-2013-6/ (accessed December 11, 2018).

Stone \& Stone (1989), Embracing Ourselves. The Voice Dialogue Manual, Nataraj Publishing, Novato, CA.

Van Knippenberg, D. and Sleebos, E. (2006), "Organizational identification versus organizational commitment: self-definition, social exchange, and job attitudes", Journal of Organizational Behavior: The International Journal of Industrial, Occupational and Organizational Psychology and Behavior, Vol. 27 No. 5, pp. 571-584.

\section{Further reading}

McDonough, W. and Braungart, M. (2010), Cradle to Cradle: Remaking the Way We Make Things, North point press, New York, NY.

\section{Corresponding author}

Dr Kyrill Goosseff can be contacted at:kyrgoo@gmail.com

For instructions on how to order reprints of this article, please visit our website: 